\title{
Stability analysis of closed-loop anaesthesia system with nonlinear uncertainty: a circle criterion approach
}

\begin{abstract}
Inter-individual variability is one of the major challenge in closed-loop control of anaesthesia. This variability could lead to stability problem and must be taken into account during the evaluation of controller. While studies have suggested that variability in nonlinear pharmacodynamics (PD) model is much higher than the linear pharmacokinetic (PK) model, most of the robust stability analyses have only considered uncertainty that exists linearly. In this study, robust stability analysis of a closed-loop anaesthesia control system on uncertainty in nonlinearity was performed. The closed-loop control system consists of a PKPD model and a PID controller that was treated as a Lur'e problem. Circle criterion was applied to determine the bound of nonlinearity that guarantees asymptotic stability of the system. Result shows that the stability bound of the controller is sufficiently large for the possible inter-variability in non-linearity among patients.
\end{abstract}

Keyword: Absolute stability; Depth of anaesthesia; Model uncertainty; Nonlinearity; PKPD model 